\title{
III. - EFFets DU Pourcentage de pommes ensilées DANS LE RÉGIME ALIMENTAIRE DU PORC, SUR LA DÉPENSE ALIMENTAIRE, LA CROISSANCE ET LES QUALITÉS DE CARCASSE
}

PAR

\author{
A. M LEROY et S. Z. ZELTER ( $\left.{ }^{1}\right)$
}

Pour résoudre le problème de la résorption des excédents de fruits à cidre et rechercher les conditions qui permettraient d'en tirer le meilleur parti possible dans l'alimentation animale, nous avons pensé que le porc pouvait être un excellent utilisateur de cet aliment essentiellement glucidique. En l'absence totale d'informations relatives à l'utilisation de pommes conservées par ensilage, nous nous sommes attachés à déterminer en particulier les limites d'emploi de celles-ci pour le porc en observant l'influence de doses croissantes de cet ensilage sur la croissance, la dépense alimentaire et la qualité de la viande produite.

\section{TECHNIQUE EXPERIMENTALE}

\section{A. - Matériel animal.}

Onze portées de 4 porcelets de races Danoise et I arge White ont été associées de façon à former des lots de 4 individus semblables par 1'origine, le sexe et le poids. Ceux-ci ont été répartis dans les $4 \times 4$ cases qui pouvaient loger I, 2, 3 ou 5 . sujets. I,es croissances individuelles et les indices moyens de consommation des unités indivisibles ainsi créées ont d'abord été déterminés au cours d'une période préliminaire de 2 I jours. Ces mesures nous ont permis de constituer selon la méthode de couples 4 groupes comparables de II porcs (voir tableau I), chaque sujet de l'un des groupes ayant son correspondant dans chacun des trois autres.

Les pourcentages de matières sèches des pommes à expérimenter ayant été fixés théoriquement à $\mathrm{I} 5,20$ et 25 , les traitements suivants ont été affectés aux divers groupes par un tirage au sort effectué à l'issue de la période d'observation.

(1) Avec l'assistance technique de B. Dupont et C. Dumar: 
TABLEAU I

Période préliminaire.

\begin{tabular}{|c|c|c|c|c|c|}
\hline Groupe & $\begin{array}{c}\text { Poids de début } \\
\text { de période }\end{array}$ & $\begin{array}{l}\text { Poids de fin } \\
\text { de période }\end{array}$ & $\begin{array}{c}\text { Gain quotidien } \\
\text { moyen }\end{array}$ & $\left|\begin{array}{c}\text { Consonmation } \\
\text { quotidieme de } \\
\text { mat. séche }\end{array}\right|$ & $\begin{array}{l}\text { Indice de } \\
\text { colsommation }\end{array}$ \\
\hline & $\mathrm{kg}$ & $\mathrm{kg}$ & $\xi$ & $\mathrm{kgr}$ & IT. F. \\
\hline $\mathrm{T}_{n} \ldots$ & $2 \mathrm{I}, \mathrm{I}$ & 33,2 & $578 \pm 30$ & $t, 42$ & 2,70 \\
\hline $15 \mathrm{E}$ & $2 \mathrm{I}, 3$ & 33,5 & $583 \div 43$ & $\mathrm{I}, 4$ & $2,7^{2}$ \\
\hline $20 \mathrm{E}$ & $2 \mathrm{I}, \mathrm{I}$ & 34,0 & $615 \pm 27$ & $\mathrm{I}, 45$ & 2,57 \\
\hline $25 \mathrm{E}$ & 20,6 & $3^{2,7}$ & $57^{6} \pm 44$ & $\mathrm{I}, 43$ & 2,67 \\
\hline
\end{tabular}

Groupe

$\mathrm{T}_{0}$ (témoin)... Régime de référence ne comjortant pas de pommes.

$\mathbf{E}_{15} \ldots . . . \ldots$ Régime expérimental I : $15 \%$ de matière sèche sous forme de pommes ensilées

$\mathrm{E}_{20} \ldots \ldots \ldots$ Régime expérimental $2: 20 \%$ de matière sèche sous forme de pommes ensilées

$\mathrm{E}_{25} \ldots \ldots \ldots$ Régime expérimental $3: 25 \%$ de matière sèche sous forme de pommes ensilées

En fait, les pourcentages réels (I6,6, 2I,2 et 26,6) ont été légèrement supérieurs à ceux que nous avions fixés à priori.

Une période de 15 jours d'accoutumance aux régimes d'expérience a précédé la période expérimentale, qui a pris fin 70 jours après, lorsque 1 e poids moyen d'un premier lot de porcs a atteint $93 \mathrm{~kg}$ environ.

$I_{\text {a }}$ pesée individuelle des animaux une fois par semaine dans des conditions identiques nous a permis de suivre l'évolution du gain de poids.

\section{B. - Alimentation.}

Trois repas journaliers (leur durée a été limitée à vingt minutes pour la ration de référence et à $3 / 4$ d'heure pour les rations expérimentales plus volumineuses du fait de la présence de pommes) ont été distribués aux animaux. Le rationnement a été adapté à l'appétit et les consommations systématiquement relevées. Un aliment de référence dit " de croissance " à I 4,30 p. roo de protides digestibles a été consommé par tous les groupes durant la période préliminaire et par le lot-témoin $\left(\mathrm{T}_{\mathbf{0}}\right)$ seulement pendant une partie de la période expérimentale jusqu'à un poids moyen de $60 \mathrm{~kg}$. Il a été remplacé, ensuite, par un autre appelé "d'engraissement " moins riche en matières azotées digestibles (I, 5 p. Ioo). I,es formules types de ces deux mélanges alimentaires témoins utilisées au cours de plusieurs expériences antérieures semblables, ont été indiquées dans nos précédentes publications $(\mathrm{I}, 2)$.

Les régimes expérimentaux comportaient : des pommes à cidre ensilées, du concentré azoté dosant 38 p. roo de protides digestibles totaux et de l'orge moulue.

L'ensilage de pommes a été préparé avec un mélange de trois variétés (Mettais, Binet doré et Longbois) et conservé depuis le 7 décembre I954 
sous une couche de marc frais de cidrerie dans une tranchée faite dans la terre battue; il a été utilisé entre le $\mathrm{I}_{5}$ février et le Io mai suivants. Au moment de sa consommation cet ensilage dosait en moyenne I I, 9 p. Ioo de matière sèche (alcool inclus), o,8 p. Ioo d'acide acétique, o, I3 p. Ioo d'acide lactique, I,I7 p. Ioo d'alcool, et était exempt d'acide butyrique.

Des quantités croissantes de pommes ainsi conservées ont été distribuées de manière à maintenir sensiblement constants dans les rations de chaque groupe, les pourcentages de matière sèche provenant de cet ensilage.

Une dose fixe $(0,500 \mathrm{~g})$ de concentré azoté (levure de distillerie 7 p.Ioo, tourteau d'arachide $65 \mathrm{p}$. Ioo, farine de viande $\mathrm{I} 2 \mathrm{p}$. roo, farine de poisson 5 p. Ioo, mélange minéral II p. IOo) a été donnée journellement à chaque porc avec les pommes. Des quantités d'orge moulue consommées à part ont permis d'adapter la distribution de nourriture à l'appétit des sujets; elles complétaient les régimes d'expérience.

Cette façon d'alimenter les animaux a permis de fournir aux groupes expérimentaux des régimes ayant des teneurs en protides digestibles, cellulose brute, phosphore et calcium équivalentes à celles du régime témoin $\left(\mathrm{T}_{0}\right)$. Les caractéristiques essentielles des aliments utilisés sont indiquées ci-après.

\section{TABLEAU II}

Caractéristiques alimentaires essentielles des régimes.

\begin{tabular}{|c|c|c|c|c|c|}
\hline & \multicolumn{5}{|c|}{ en $g$. pour mille d'aliment brut } \\
\hline & \multicolumn{2}{|c|}{ Régime de rèférence } & \multicolumn{3}{|c|}{ Règime d'expérience } \\
\hline & $\begin{array}{c}\text { Aliment } \\
\text { de croissance }\end{array}$ & $\begin{array}{l}\text { Aliment } \\
\text { d'expérience }\end{array}$ & $\begin{array}{l}\text { Ensilage } \\
\text { de pommes }\end{array}$ & $\begin{array}{c}\text { Concentré } \\
\text { azoté }\end{array}$ & $\begin{array}{l}\text { orge } \\
\text { moulue }\end{array}$ \\
\hline Matiére sèche....... & 867 & 876 & $\stackrel{\text { IIg }}{\text { (alcool inclus) }}$ & 887 & 860 \\
\hline $\begin{array}{l}\text { Cellulose weende } \ldots \ldots \ldots \\
\text { Protides totaux digest.... } \\
\text { Ca } \ldots \ldots \ldots \ldots \ldots \ldots \ldots \ldots \ldots \\
\text { P } \ldots \ldots \ldots \ldots \ldots \ldots \ldots \ldots\end{array}$ & $\begin{array}{c}53 \\
143 \\
19 \\
9,3\end{array}$ & $\begin{array}{c}5^{6} \\
1^{5} 5 \\
9,4 \\
5,5\end{array}$ & $\begin{array}{c}\text { I3 } \\
0 \\
0,6 \\
0,2\end{array}$ & $\begin{array}{c}43 \\
380 \\
40 \\
13,4\end{array}$ & $\begin{array}{c}48 \\
73 \\
4,2 \\
3,6\end{array}$ \\
\hline $\begin{array}{r}\text { Valeur nutritive en U. F. } \\
\text { par } \mathrm{kg} . \ldots \ldots \ldots \ldots \ldots \ldots\end{array}$ & 0,948 & 0.964 & Inconnue & $0,95^{2}$ & $I, 000$ \\
\hline
\end{tabular}

Une distribution bi-hebdomadaire d'huile de poisson apportait par jour, à chaque animal, 2500 U. I. de vitamine A et 330 U. I. de vitamine $\mathrm{D}_{2}$. 


\section{RÉSULTATS}

\section{A. - Consommation de nourriture.}

Les animaux ont mis près de quinze jours à s'habituer progressivement aux pourcentages prévus de matière sèche de pommes ensilées. Plus les pourcentages étaient importants, plus s'est trouvée réduite l'appétence des régimes. Pour obliger les porcs à ingérer la totalité des pommes qui leur étaient données, il a fallu rationner sévèrement l'orge. Durant la première semaine de la période expérimentale, les quantités journalières de pommes absorbées dans les groupes $\mathrm{E}_{15}, \mathrm{E}_{20}$ et $\mathrm{E}_{25}$ ont été respectivement de $2,0,2,6$, et $3,6 \mathrm{~kg}$; elles se sont élevées à $3,4,4,2$ et $4,8 \mathrm{~kg}$ au cours de la dernière.

Le manque d'appétence des rations à base de pommes conservées par ensilage a entraîné un abaissement sensible de la consommation de matière sèche, qui a été, de ce fait, nettement en dessous de celle du groupe-témoin, comme le montre le tableau III.

'TABLEAU III

Période expérimentale.

Consommations moyennes journalières par porc.

\begin{tabular}{|c|c|c|c|c|}
\hline Groupe & $\mathrm{T}_{0}$ & $\mathrm{E}_{15}$ & $\mathrm{E}_{20}$ & $E_{25}$ \\
\hline Pourcentage réel de matière sèche de pommes dans la ration & 0,0 & 16,6 & $2 \mathrm{I}, 2$ & 26,6 \\
\hline Matières azotées totales digestibles $\%$ de matière sèche.... & 14,04 & 14,29 & r $4,9 \mathrm{r}$ & $\mathrm{I} 4,6 \mathrm{I}$ \\
\hline Cellulose Weende $\%$ de matière sèche $\ldots \ldots \ldots \ldots \ldots$ & 6,34 & $6,4 \mathrm{I}$ & 6,69 & 7,03 \\
\hline $\mathrm{Ca} \%$ de matière sèche $\ldots \ldots \ldots \ldots \ldots \ldots \ldots$ & I,34 & $\mathrm{r}, 45$ & 1,60 & 1,56 \\
\hline $\mathrm{P} \%$ de matière sèche & 0,73 & 0,64 & 0,66 & \\
\hline Matière sèch & 2674 & 2063 & 1922 & I 868 \\
\hline m. s. de l'alime & 2674 & o & & \\
\hline ensilées (alcool inclus) (g) & o & 337 & 408 & 496 \\
\hline m. s. de concentré azoté (g) & $\circ$ & 477 & $49 I$ & 477 \\
\hline in. s. d'orge (g) & $\circ$ & I 249 & I 023 & 895 \\
\hline
\end{tabular}

\section{B. - Croissance, dépense alimentaire et qualité de carcasse.}

Les données des tableaux IV, $\mathrm{V}$ et la figure I permettent de comparer l'influence des régimes contenant des pourcentages variables de pommes ensilées sur la croissance, la dépense alimentaire, l'efficacité nutritive et les qualités de carcasse des divers groupes.

L'inégalité du rythme de croissance n'a pas permis d'abattre en fin d'expérience dans chaque groupe, un nombre élevé et égal de porcs de poids comparable. Par ce fait, nous avons dû limiter 1'épreuve d'abattage aux seuls sujets dont le poids a atteint 90 à $105 \mathrm{~kg}$. Ces derniers ont été au nombre de 4 dans les groupes $T_{0}$ et $\mathrm{E}_{15}$, et de deux dans ceux $\mathrm{E}_{20}$ et $\mathrm{E}_{25}$. Les mensurations effectuées sur les carcasses sont indiquées dans le tableau V. Elles permettent d'apprécier le rendement de viande et l'état 
TABLEAU IV

Croissance et dépense alimentaire (période expérimentale).

\section{Groupe}

m. s. pommes \% dem. s. ingérée ......

Poids moyen initial $(\mathrm{kg}) \ldots \ldots \ldots \ldots \ldots$

Poids moyen final $(\mathrm{kg})$

Gain moyen quotidien (g) ...........

Indice de consommation exprimé en $\mathrm{kg}$ de matière sèche ..............

Quotient de l'efficacité nutritive de la matière sèch: des régimes d'expérience par rapport à celui de la matiêre sèche du régime de référence $\ldots \ldots \ldots \ldots \ldots$

Indice de consommation exprimé en unités fourragère: (déduction faite ce la matière sèche de pommes)

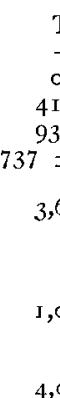

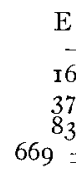

$\mathrm{E}_{15}$

16,6

37,4

$669 \pm 24$

3,08

I,20

I, I 2

2,98

2,96

$+0,504$

$+0,703$

$\mathrm{E}_{20}$

E 25 (alcool inclus)

Poids moyen

$$
\text { Kgs }
$$

too

COURBes DE CROISSANCE, MOYenNe

95

90

80

70

55

55

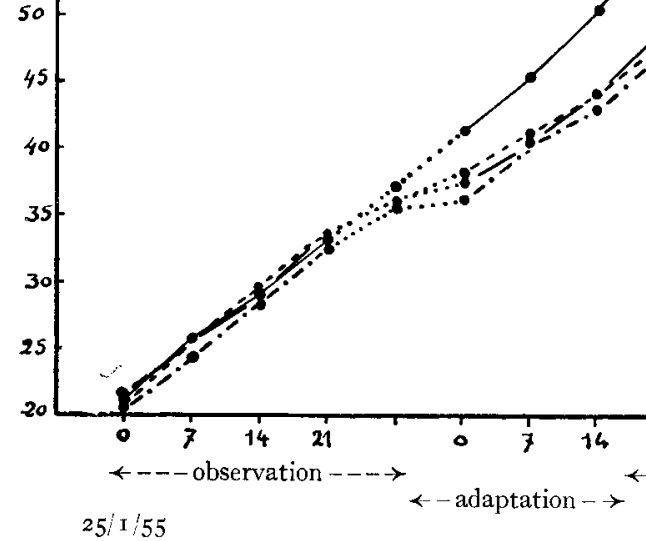

FIG. I 
d'engraissement sous l'influence des divers types de régimes. Seules peuvent être comparées valablement entre elles les observations faites sur les lots $T_{0}$ et $E_{15}$ qui ont eu un même nombre de sujets sacrifiés à des poids sensiblement voisins. Les résultats des lots $E_{20}$ et $E_{25}$ ne sont rapportés qu'à titre indicatif, car ils sont moins nombreux et concernent des animaux abattus à un poids inférieur.

\section{TABLEAU $\mathrm{V}$}

Résultats individuels de l'éprenve d'abatage.

\begin{tabular}{|c|c|c|c|c|c|c|c|c|c|}
\hline \multirow[t]{2}{*}{ Groupe } & \multirow{2}{*}{$\begin{array}{l}\text { No du } \\
\text { sujet }\end{array}$} & \multirow[t]{2}{*}{ Races } & \multirow[t]{2}{*}{ Sexe } & \multirow{2}{*}{$\begin{array}{l}\text { Poids } \\
\text { d'aba- } \\
\text { tage kg }\end{array}$} & \multirow{2}{*}{$\begin{array}{c}\text { Croissance } \\
\text { moyenne } \\
\text { journalière } \\
\mathrm{g}\end{array}$} & \multirow{2}{*}{$\begin{array}{l}\text { Rende- } \\
\text { ment \% } \\
\text { (carcasse } \\
+ \text { têtes) }\end{array}$} & \multicolumn{2}{|c|}{$\begin{array}{l}\text { Épaisseur } \\
\text { du lard } \\
\text { en } \mathrm{mm}\end{array}$} & \multirow{2}{*}{$\begin{array}{l}\text { Catégorie } \\
\text { commer- } \\
\text { ciale }\end{array}$} \\
\hline & & & & & & & Dos & Rein & \\
\hline $\mathrm{T}_{0}$ & $\begin{array}{l}16 \\
29 \\
44 \\
56\end{array}$ & $\begin{array}{l}\text { Dan. } \\
\text { L. W. Y. } \\
\text { L. W. Y. } \\
\text { I. W. Y. }\end{array}$ & $\begin{array}{l}F \\
M \\
M \\
M\end{array}$ & $\begin{array}{r}103,0 \\
92,0 \\
105,2 \\
101,2\end{array}$ & $\begin{array}{l}780 \\
740 \\
830 \\
760\end{array}$ & $\begin{array}{l}81,6 \\
76,1 \\
78,9 \\
79,0\end{array}$ & $\begin{array}{l}32 \\
36 \\
40 \\
44 \\
\end{array}$ & $\begin{array}{l}28 \\
31 \\
39 \\
45 \\
\end{array}$ & $\begin{array}{l}\text { belle coupe } \\
\text { belle coupe } \\
\text { gras } \\
\text { gras }\end{array}$ \\
\hline Moyenne.. & & & & 100,3 & $\overline{777}$ & 78,9 & $\overline{38}$ & 36 & \\
\hline $\mathrm{E}_{15}$ & $\begin{array}{l}\text { I } 2 \\
50 \\
47 \\
55\end{array}$ & $\begin{array}{l}\text { Dan. } \\
\text { L. W. Y. } \\
\text { L. W. Y. } \\
\text { L. W. Y. }\end{array}$ & $\begin{array}{l}\mathrm{F} \\
\mathrm{F} \\
\mathrm{F} \\
\mathrm{M}\end{array}$ & $\begin{array}{r}100,4 \\
96,4 \\
93,4 \\
99,0 \\
\end{array}$ & $\begin{array}{l}710 \\
730 \\
680 \\
770 \\
\end{array}$ & $\begin{array}{l}78,7 \\
76,8 \\
77: \mathbf{I} \\
77,8 \\
\end{array}$ & $\begin{array}{l}32 \\
31 \\
36 \\
30 \\
\end{array}$ & $\begin{array}{l}3 \mathrm{I} \\
27 \\
30 \\
26 \\
\end{array}$ & $\begin{array}{l}\text { belle coupe } \\
\text { belle coupe } \\
\text { belle coupe } \\
\text { complet }\end{array}$ \\
\hline Moyenne.. & & & & 97,3 & 722 & $\overline{77,6}$ & 32 & 29 & \\
\hline$E_{20}$ & $\begin{array}{r}2 \\
45\end{array}$ & $\begin{array}{l}\text { Dan. } \\
\text { L. W. Y. }\end{array}$ & $\begin{array}{l}\mathrm{M} \\
\mathrm{M}\end{array}$ & $\begin{array}{r}100,6 \\
91,2 \\
\end{array}$ & $\begin{array}{l}7 \mathrm{co} \\
660 \\
\end{array}$ & $\begin{array}{l}78,5 \\
75,7 \\
\end{array}$ & $\begin{array}{l}28 \\
31 \\
\end{array}$ & $\begin{array}{l}28 \\
22 \\
\end{array}$ & $\begin{array}{l}\text { complet } \\
\text { complet }\end{array}$ \\
\hline Moyenne.. & & & & 95,9 & 680 & 77,1 & 30 & 25 & \\
\hline $\mathrm{E}_{20} \quad \ldots$ & $\begin{array}{r}I \\
46\end{array}$ & $\begin{array}{l}\text { Dan. } \\
\text { L. W. Y. }\end{array}$ & $\begin{array}{l}\mathrm{M} \\
\mathrm{M}\end{array}$ & $\begin{array}{l}90,6 \\
92,6\end{array}$ & $\begin{array}{l}640 \\
670 \\
\end{array}$ & $\begin{array}{l}77,3 \\
76,7 \\
\end{array}$ & $\begin{array}{l}30 \\
3 \mathrm{I} \\
\end{array}$ & $\begin{array}{l}20 \\
20 \\
\end{array}$ & $\begin{array}{l}\text { complet } \\
\text { complet }\end{array}$ \\
\hline Moyenne... & & & & $9 \mathrm{I}, 6$ & $\overline{655}$ & $\overline{77,0}$ & $\overrightarrow{3 I}$ & $\overline{20}$ & \\
\hline
\end{tabular}

\section{DISCUSSION DES RÉSULTATS}

\section{a) Influence des pommes ensilées sur la croissance.}

Rappelons que notre dispositif expérimental a été fondé sur la méthode de couples. Ceci rend possible la comparaison entre croissances moyennes individuelles des sujets des groupes expérimentaux $\left(\mathrm{E}_{15}, \mathrm{E}_{20}\right.$ et $\mathrm{F}_{25}$ ) et celles de leurs homologues du groupe témoin $\left(\mathrm{T}_{0}\right)$, comparaison qui fait ressortir que les porcs dont le régime renfermait $16,6,21,2$ et 26,6 p. Ioo de matière sèche sous forme de pommes ensilées accusent une croissance retardée respectivement de 9,I, 2I,3 et 20,4 p. Ioo. Ces écarts, qui sont significativement en défaveur des rations expérimentales (voir tableau VI) prouvent que le ralentissement du gain de poids s'accentue avec l'élévation du pourcentage de pommes introduites dans le régime. 
TABLEAU VI

Ecarts moyens entre croissances individuelles

des sujets du groupe témoin et des groupes expérimentaux.

\begin{tabular}{|c|c|c|c|c|c|c|}
\hline \multirow{2}{*}{$\begin{array}{c}\text { Groupes } \\
\text { comparés }\end{array}$} & \multicolumn{3}{|c|}{ Préexpérience } & \multicolumn{3}{|c|}{ Expérience } \\
\hline & $\begin{array}{c}\text { Différence absolue } \\
(\mathrm{g})\end{array}$ & $\begin{array}{l}\text { Différence } \\
\text { relative (\%) }\end{array}$ & $\underset{t}{\text { Valeur }}$ & $\begin{array}{l}\text { Différence absolue } \\
(\mathrm{g})\end{array}$ & $\left|\begin{array}{c}\text { Différence } \\
\text { relative }(\%)\end{array}\right|$ & $\begin{array}{c}\text { Valeur } \\
t\end{array}$ \\
\hline $\begin{array}{l}\mathrm{T}_{o}-\mathrm{E}_{15} \ldots \ldots \\
\mathrm{T}_{o}-\mathrm{E}_{20} \ldots \\
\mathrm{T}_{o}-\mathrm{E}_{25} \ldots\end{array}$ & $\begin{array}{r}5,1 \pm 16,9 \\
-37,3 \pm 20,8 \\
+\quad 1,6 \pm 29,8\end{array}$ & $\begin{array}{r}-0,9 \\
-6,5 \\
+\quad 0,3\end{array}$ & $\begin{array}{l}0,38 \\
1,79 \\
0,05\end{array}$ & $\begin{array}{r}+67,4 \pm 27,5 \\
+\quad \text { I } 56,7 \pm 47,3 \\
+\quad \mathrm{I} 50, \mathrm{I} \pm 42,7\end{array}$ & $\begin{array}{l}+\quad 9, \mathbf{I} \\
+\quad 2 \mathbf{I}, 3 \\
+\quad 20,4\end{array}$ & $\begin{array}{l}2,45 \\
3,32 \\
3,52\end{array}$ \\
\hline
\end{tabular}

En consultant le tableau III, on remarque que :

- les teneurs de toutes les rations consommées, en protides digestibles, en cellulose, en calcium et en phosphore étaient pratiquement équivalentes ;

- les doses journalières moyennes de calcium $(27,4 \mathrm{~g})$ et de phosphore (II,8 g) absorbées par les porcs du groupe $\mathrm{E}_{25}$ qui a ingéré la plus faible quantité de matière sèche, dépassaient largement les minima requis selon les normes classiques;

- la consommation quotidienne moyenne de nourriture s'abaissait d'autant plus que le pourcentage de pommes incorporées dans le régime augmentait : dans les groupes $\mathrm{F}_{15}, \mathrm{~F}_{20}$ et $\mathrm{F}_{125}$, les absorptions respectives de matière sèche ne représentent que 77,4 p. I00, 72,o p. Ioo et 70,0 p. Ioo de celle enregistrée dans le groupe $T_{0}$, qui ne recevait pas de pommes. Ce dernier fait constitue incontestablement la cause essentielle de l'abaissement du gain de poids des animaux dont les rations contenaient 1'aliment expérimenté.

Sachant que la masse de liquide est susceptible de réduire la capacité d'absorption de'nourriture chez le porc, il est vraisemblable que si les pommes distribuées avaient été moins aqueuses $(88$, I p. Ioo d'humidité) et n'avaient pas contribué à augmenter exagérément le volume des rations, ainsi que l'inappétence, les ingestions de matière sèche des sujets expérimentaux se seraient maintenues à un niveau plus élevé ; dans ce cas, la croissance aurait donc été meilleure. On peut, dès lors, supposer qu'avec une ration à $x 6,6 \mathrm{p}$. roo de matière sèche de fruits plus riches en substance nutritive, le gain de poids ne serait pas significativement différent de celui observé avec le régime témoin.

Si l'on compare, d'autre part, les résultats de l'épreu ve d'abattage (tableau V), on constate pour des porcs à croissance équivalente, sacrifiés au même poids, que la présence dans le régime, de pommes conservées par ensilage, améliore nettement la qualité de la carcasse. 


\section{b) Effet sur l'indice de consommation.}

I'efficacité nutritive des régimes expérimentat1x paraît, en revanche, supérieure à celle du régime exempt de pommes. Pour les groupes $\mathrm{E}_{15}$, $\mathrm{F}_{20}$ et $\mathrm{E}_{25}$, les indices de consommation de matière sèche sont, respectivement, de 3,08, 3,33 et 3,I $8 \mathrm{~kg}$, contre 3,68 $\mathrm{kg}$ pour le groupe témoin $\mathrm{T}_{\mathbf{0}}$ (tableau IV). L'incorporation de cet aliment semble donc avoir amélioré l'utilisation énergétique des éléments des rations expérimentales, puisque l'indice de consommation des animaux qui les ont reçues a fléchi de 9,4 p. Ioo au moins $\left(\mathrm{E}_{120}\right)$ et de $I 6,6$ p. Ioo au plus $\left(\mathrm{E}_{15}\right)$. Ceci laissant supposer une valeur énergétique particulièrement élevée pour la matière sèche de pommes, il nous a paru intéressant de la calculer à partir de nos mesures expérimentales, selon une méthode indirecte déjà employée par nous dans d'autres études analogues $(I, 2)$. D'après ce calcul, la valeur nutritive $\mathrm{du} \mathrm{kg}$ de matière sèche de pommes consommées par les groupes $E_{15}$, $\mathrm{E}_{20}$ et $\mathrm{E}_{25}$ s'élèverait respectivement à 2,I $8, \mathrm{I}, 77$ et $\mathrm{x}, 78$ unités fourragères. Ces données, particulièrement fortes, surprennent d'autant plus qu'elles concernent un aliment qui a perdu, au cours de la fermentation en silo, la majeure partie de son sucre dont l'assimilabilité est très élevée. On doit leur opposer, d'une part le résultat nettement plus faible (I,I 86 u. f.), observé en digestibilité directe chez le mouton pour des pommes à l'état frais (voir mémoire II), et d'autre part, la valeur maximum (I,47 u. f.) qui découlerait de la composition chimique de l'aliment étudié si l'on admettait que l'utilisation digestive de ses constituants était intégrale chez le porc.

On peut évidemment supposer que les valeurs discutées aient pu se trouver "surévaluées ", car elles ont été déduites d'un indice de consommation hypothétique admis pour un lot témoin qui, ayant produit des carcasses plus grasses que les groupes nourris avec des pommes, a eu des dépenses énergétiques plus élevées. A moins que leur explication ne doive être recherchée partiellement dans une présence éventuelle, dans nos pommes qui ont subi une intense fermentation alcoolique, de facteurs non identifiés, stimulant la croissance (?), comme l'ont signalé récemment ComBs et coll. (3) pour des solubles déshydratés provenant de la fermentation, en distillerie de mélasse et de grains, et Noland et coll. (4) pour d'autres résidus secs de fermentation. Notons à ce sujet, une observation empirique rapportée par plusieurs éleveurs qui, ayant distribué du cidre de pommes à des veaux, ont remarqué une très nette amélioration du développement corporel de ces animaux. Mais notre supposition est une simple hypothèse qui nécessite de nouvelles recherches. 


\section{RÉSUMÉ ET CONCLUSIONS}

Un essai d'utilisation de pommes à cidre conservées par ensilage sous une couche de marc de cidrerie, a été fait avec 4 groupes comparables de II porcelets sevrés. Cet aliment renfermait II,9 p. roo de substance sèche avec I, I 7 p. Ioo d'alcool.

Des taux d'incorporation, dans la ration, de $0,16,6,2 \mathrm{I}, 2$ et $26,6 \mathrm{p}$. Ioo de matière sèche sous forme de pommes ont été expérimentés ; la croissance, l'indice de consommation et la qualité de carcasse servaient de critères.

Les animaux ont parfaitement toléré l'aliment étudié, dont la trop grande richesse en eau a eu pour conséquence un abaissement du niveau de consommation de nourriture.

Dans nos conditions expérimentales, la croissance a été très satisfaisante, bien que légèrement, mais significativement diminuée $(-9, \mathrm{I}$ p. Ioo) avec un régime comportant 16,6 p. Ioo de matière sèche en provenance d'ensilage de pommes. En présence de pourcentages plus élevés, le retard de croissance s'est fortement accentué ( $2 \mathrm{r}, 3$ p. Ioo). Le faible ralentissement de gain de poids qu'entraîne une ingestion quotidienne de $16,6 \mathrm{p}$. Ioo de matière sèche à l'état de pommes ensilées, se trouve compensé par une amélioration sensible de l'indice de consommation et de la qualité de carcasse. Il semble donc souhaitable de limiter l'incorporation de cet aliment dans le régime du porc à un taux légèrement inférieur.

L'adoption d'un tel régime pour le porc fermier des régions cidricoles présente une solution rentable du problème des excédents et des rebuts de la production du verger, dont elle permettrait d'améliorer le revenu. Elle offre, de plus, la possibilité de réduire substantiellement la fabrication d'alcool de fruits et de comprimer le coût de l'engraissement porcin.

\section{RÉFÉRENCES BIBLIOGRAPHIQUES}

(I) Leroy (A. M.), Zeiter (S. Z.), FÉvrier (R.). - Ann. de Zoot., I952, 1, $n^{0} \mathrm{I}, 87$.

(2) LeRoy (A. M.), Zeiter (S. Z.). - Ann. de Zoot., I954, 3, 110 2, IO9.

(3) Combs (G. F.), Arscott (G. H.), Jones (H. L.). - Poultry Science, I954, 33, no $\mathrm{I}, 7 \mathrm{I}$.

(4) Noland (P. R.), Stephenson (E. L.), Diggs (B.).-J. of Anim. Sci., I955, $14, \mathrm{n}^{\circ} 3,628$. 